\title{
THE STRUCTURE OF ASSOCIATOR DEPENDENT RINGS
}

BY

E. KLEINFELD, F. KOSIER, J. MARSHALL OSBORN, AND D. RODABAUGH(1)

Introduction. A number of authors have studied rings $A$ that satisfy an identity of the form:

$$
\begin{aligned}
\alpha_{1}(x, y, z)+\alpha_{2}(y, z, x)+ & \alpha_{3}(z, x, y) \\
& +\alpha_{4}(x, z, y)+\alpha_{5}(z, y, x)+\alpha_{6}(y, x, z)=0
\end{aligned}
$$

for fixed $\alpha_{i}$ in some field of scalars and all $x, y, z$ in $A$ where the associator is defined by $(x, y, z)=(x y) z-x(y z)$. Kleinfeld has called rings $A$ satisfying (1) and

$$
(x, x, x)=0
$$

"associator dependent rings" and we shall adopt this convention [6]. It is immediate that the right and left alternative rings, the flexible rings, the rings of type $(\gamma, \delta)$ and the anti-flexible rings all belong to the class of associator dependent rings.

Linearizing (2) yields (1) with $\alpha_{i}=1$ for $i=1, \cdots, 6$. Since (2) is certainly not strong enough to give a structure theory, we assume further that $A$ satisfies an identity of the form (1) which is not implied by (2). We show in $\S 2$ that $A$ must then satisfy one of the following identities:

$$
\begin{aligned}
\alpha(y, x, x)-(\alpha+1)(x, y, x)+(x, x, y) & =0, \alpha \text { a fixed scalar, } \\
(y, x, x) & =(x, y, x), \\
(x, y, z)+(y, z, x)+(z, x, y) & =0 .
\end{aligned}
$$

W. E. Coppage is studying (5) in his Ph. D. dissertation $\left({ }^{2}\right)$. We note that any ring satisfying $(y, x, x)=(x, y, x)$ is anti-isomorphic to a ring satisfying

Presented to the Society, March 12, 1962 under the title $A$ generalized associator identity, and, on August 29, 1962 under the title Nonassociative algebras satisfying identities of degree three; received by the editors July 30, 1962 and, in revised form, March 15, 1963.

(1) The research of the first three authors was supported by the U. S. Army Research Office (Durham), the Wisconsin Alumni Research Foundation, and Grant G-19052 of the National Science Foundation, respectively.

(2) Rings satisfying (5) are Lie-admissible and some literature already exists. For some interesting results on Lie-admissible rings see P. J. Laufer and M. L. Tomber, Some Lie-admissible rings, Canad. J. Math. 14 (1962), 287-292. We also note that commutative rings and hence, Jordan rings as well as Lie rings satisfy (5). 
$(x, x, y)=(x, y, x)$ which is (3) with $\alpha=0$. Hence, we concentrate our attention on (3). We find that rings $A$ satisfying (2) and (3) with $\alpha \neq 1,-\frac{1}{2},-2$, have a Peirce decomposition $A=A_{11}+A_{10}+A_{01}+A_{00}$ with respect to an idempotent $e$ of $A$. The main results are then:

(a) Any simple ring $A$ satisfying (2) and (3) with $\alpha \neq 1,-\frac{1}{2},-2$, and possessing an idempotent $e$ such that $A_{10}+A_{01} \neq 0$ is either associative or a Cayley-Dickson algebra over its center( $\left.{ }^{3}\right)$.

(b) Let $A$ be a power-associative finite-dimensional algebra satisfying (2) and (3) with $\alpha \neq-1$. If $A$ is semi-simple ( $A$ has zero nil radical) then $A$ has a unity element and is the direct sum of simple algebras.

The result (a) can be improved if we also exclude the value $\alpha=-1$. In this instance we may replace the condition that $A$ have an idempotent $e$ such that $A_{10}+A_{01} \neq 0$ by the condition that $A$ have an idempotent $e \neq 1$.

The methods of proof are those employed by Kleinfeld [6], Kokoris [7], and Kosier [9]. Namely, one uses the Peirce decomposition to construct the ideal $L=A_{10} A_{01}+A_{10}+A_{01}+A_{01} A_{10}$ and then, invoking the simplicity one sees that $A_{11}=A_{10} A_{01}$ and $A_{00}=A_{01} A_{10}$. Using these representations of $A_{11}$ and $A_{00}$ we prove that they are associative subrings of $A$. From this it can then be shown that $A$ is alternative.

2. Main section. We begin our study by reducing (1) with the aid of (2) to three possibilities.

THEOREM 1. Let $A$ be a ring of characteristic not 2 or 3 which satisfies an identity of the form (1) (which is not implied by (2)) and identity (2). Then A satisfies either (3), (4), or (5).

Proof. We first linearize $(x, x, x)=0$ to obtain

$$
(x, y, z)+(y, z, x)+(z, x, y)+(x, z, y)+(z, y, x)+(y, x, z)=0 .
$$

By performing the three even permutations on $x, y, z$ in (1) we obtain three equations which when added give

$$
\begin{aligned}
& \left(\alpha_{1}+\alpha_{2}+\alpha_{3}\right)[(x, y, z)+(y, z, x)+(z, x, y)] \\
& \quad+\left(\alpha_{4}+\alpha_{5}+\alpha_{6}\right)[(x, z, y)+(z, y, x)+(y, x, z)]=0 .
\end{aligned}
$$

If $\left(\alpha_{1}+\alpha_{2}+\alpha_{3}\right) \neq\left(\alpha_{4}+\alpha_{5}+\alpha_{6}\right)$, then (6) and (7) imply (5). Hence, assume $\alpha_{1}+\alpha_{3}+\alpha_{2}=\alpha_{4}+\alpha_{5}+\alpha_{6}$. Now substitute $z=x$ in (1) as well as in the equation resulting from (1) by the permutation ( $x y z)$. Then if neither of these yields an identity of the form (3) or (4) we have $\alpha_{3}+\alpha_{4}=\alpha_{1}+\alpha_{5}=\alpha_{2}+\alpha_{6}=\gamma$ and $\alpha_{2}+\alpha_{5}=\alpha_{3}+\alpha_{6}=\alpha_{1}+\alpha_{4}=\delta$. Thus $3 \gamma=\alpha_{1}+\alpha_{2}+\alpha_{3}+\alpha_{4}+\alpha_{5}+\alpha_{6}=3 \delta$, or $\gamma=\delta$. But then the assumption that $\alpha_{1}+\alpha_{2}+\alpha_{3}=\alpha_{4}+\alpha_{5}+\alpha_{6}$ would

(3) See the last paragraph of $\S 2$ for results on the excluded values $a=1,-1 / 2,-2$. 
imply that all the $\alpha_{i}$ are equal, which is impossible since we are assuming that (2) does not imply (1). In fact it can be shown that any identity which implies (5) but is not equivalent to it must imply (3) or (4).

THEOREM 2. Let $A$ be a ring satisfying (2) and (3) with $\alpha \neq 1,-\frac{1}{2},-2$. If $e$ is an idempotent of $A$ then $A$ has the Peirce decomposition $A=A_{11}+A_{10}+A_{01}+A_{00}$ where $x \in A_{i j}$ if and only if ex $=i x$, xe $=j x$; $i, j=0,1$. Moreover, the sum of the subgroups $A_{i j}$ is $\operatorname{direct}\left({ }^{4}\right)$.

Proof. It is easily seen that the theorem is valid if and only if $(e, e, x)=(e, x, e)=(x, e, e)=0$ for all $x \in A$. In any ring with an idempotent $e$ and arbitrary $x$ we have $(e, e, e x)=e(e x)-e(e(e x))=e(e, e, x)$. Substituting $x=e, y=e x$, and $x=e, y=x, z=e$ in (3) and (6) respectively and solving simultaneously we see that $(2 \alpha+1)(e x, e, e)=(2 \alpha+1) e(x, e, e)$. Since $2 \alpha+1 \neq 0$ we have $(e x, e, e)=e(x, e, e)$. Similarly one can show that $(x e, e, e)$ $=(x, e, e) e$ and $(e, x e, e)=(e, x, e) e$. Now, in an arbitrary ring

$$
(e x, e, e)-(e, x e, e)+(e, x, e)=e(x, e, e)+(e, x, e) e
$$

may be verified. However, using the above relations we may reduce this to $(e, x, e)=2(e, x, e) e$. Since this relation also holds in any ring anti-isomorphic to $A$, we see that $A$ also satisfies $(e, x, e)=2 e(e, x, e)$. Setting $a=(e, x, e)$ these relations become $a=2 e a=2 a e$. However, $4(e, a, e)=4(e a) e-4 e(a e)$ $=2 a e-2 e a=0$. Substituting $x=z=e, y=a$ in both (3) and (6) and solving simultaneously yields $(e, e, a)=0$. But then $0=4(e, e, a)=4 e a-4 e(e a)$ $=2 a-2 e a=2 a-a=a$. Then (6) implies that $(e, e, x)=-(x, e, e)$ while (3) yields $(e, e, x)=-\alpha(x, e, e)$. Since $\alpha \neq 1$, we have $(e, e, x)=(e, x, e)$ $=(x, e, e)=0$.

We show next that the following relations hold in $A$.

$$
\begin{gathered}
\alpha[(y, x, z)+(y, z, x)]-(\alpha+1)[(x, y, z)+(z, y, x)]+[(x, z, y)+(z, x, y)]=0, \\
(\alpha-1)[(x, z, y)+(z, x, y)]=-(2 \alpha+1)[(x, y, z)+(z, y, x)], \\
(\alpha-1)[(y, x, z)+(y, z, x)]=(\alpha+2)[(x, y, z)+(z, y, x)], \\
(2 \alpha+1)[(y, x, z)+(y, z, x)]=-(\alpha+2)[(x, z, y)+(z, x, y)] .
\end{gathered}
$$

Identity (8) is obtained by linearizing (3). We obtain (9) by subtracting $\alpha$ times (6) from (8), while (10) is a consequence of subtracting (6) from (8). If we add $(\alpha+1)$ times $(6)$ to $(8)$ the result is $(11)$.

We introduce the convention that any symbol $x_{i j}$ with two subscripts is assumed to represent an element of the subgroup $A_{i j}$ with the same subscripts. We now develop some of the properties of the multiplication of the subgroups $A_{i j}$.

THEOREM 3. Let $A$ be a ring satisfying (2) and (3) with $\alpha \neq 1,-\frac{1}{2},-2$. Further we suppose $A$ has characteristic prime to 6. If $\alpha \neq 0$ or -1 ,

(4) We assume throughout the remainder of this section that $a$ is an operator on the additive structure of $A$ and that $\delta x=0$ for $x \in A$ implies that $x=0$ whenever $\delta=2,3, a-1, a+2$, or $2 a+1$. 
products satisfy the following relations: $A_{i i}^{2} \subseteq A_{i i}, A_{i i} A_{j j}=0$, $A_{i j}^{2} \subseteq A_{j i}, x_{i j}^{2}=0, A_{i j} A_{j i} \subseteq A_{i i}, A_{i j} A_{j j} \subseteq A_{i j}, A_{j j} A_{i j}=0, A_{i i} A_{i j} \subseteq A_{i j}$, and $A_{i j} A_{i i}=0$, where $i=0$ or 1 and $j=1-i$. If $\alpha=-1$, then all of these relations hold except for the first one, which must be replaced by $A_{i i}^{2} \subseteq A_{i i}+A_{j j}$ and $x_{i i}^{2} \in A_{i i}$. If $\alpha=0$ all the relations of the case $\alpha \neq 0$ or -1 hold except for the last two which must be replaced by $A_{i i} A_{i j} \subseteq A_{i j}+A_{j j}$, $A_{i j} A_{i i} \subseteq A_{j j}$, and $\left(x_{i i} y_{i j}-y_{i j} x_{i i}\right) \in A_{i j}$.

Proof. Let us consider products of the form $x_{i i} y_{i i}$. It is immediate that $\left(x_{i i}, e, y_{i i}\right)=\left(y_{i i}, e, x_{i i}\right)=0$, so that setting $x=x_{i i}, y=e, z=y_{i i}$ in (9) and (10) gives $\left(x_{i i}, y_{i i}, e\right)=-\left(y_{i i}, x_{i i}, e\right)$ and $\left(e, x_{i i}, y_{i i}\right)=-\left(e, y_{i i}, x_{i i}\right)$. Then substituting $x=x_{i i}, y=y_{i i}, z=e$ in (11) yields $(2 \alpha+1)\left(y_{i l}, x_{i i}, e\right)=-(\alpha+2)\left(e, x_{i i}, y_{i i}\right)$. $\operatorname{But}\left(y_{i i}, x_{i i}, e\right) \in A_{10}+A_{00}$ while $\left(e, x_{i i}, y_{i i}\right) \in A_{01}+A_{00}$. Thus $(2 \alpha+1)\left(y_{i i}, x_{i i}, e\right)$ $=-(\alpha+2)\left(e, x_{i i}, y_{i i}\right) \in A_{00}$. But then for $i=1\left(e, y_{11}, x_{11}\right)=-\left(y_{11}, x_{11}, e\right)$ $=-\left(e, x_{11}, y_{11}\right)=\left(x_{11}, y_{11}, e\right)$. Hence $(\alpha+2)\left(e, x_{11}, y_{11}\right)=-(2 \alpha+1)\left(e, x_{11}, y_{11}\right)$ so that $3(\alpha+1)\left(e, x_{11}, y_{11}\right)=0$. If $\alpha \neq-1$, we have $\left(e, x_{11}, y_{11}\right)=\left(x_{11}, y_{11}, e\right)=0$, and this implies $A_{11}^{2}=A_{11}$. If $\alpha=-1$ we see that $\left(x_{11}, y_{11}, e\right)=-\left(y_{11}, x_{11}, e\right)$ and $\left(e, y_{11}, x_{11}\right)=-\left(e, x_{11}, y_{11}\right)$ yield $e\left(x_{11} y_{11}+y_{11} x_{11}\right)=\left(x_{11} y_{11}+y_{11} x_{11}\right) e$ $=x_{11} y_{11}+y_{11} x_{11}$ while $\left(e, x_{11}, y_{11}\right)$ and $\left(x_{11}, y_{11}, e\right)$ are elements of $A_{00}$. Thus $x_{11} y_{11} \in A_{11}+A_{00}$. In a similar manner we find that $A_{00}^{2} \subseteq A_{00}$ except possibly when $\alpha=-1$ and then we have $A_{00}^{2} \subseteq A_{11}+A_{00}$ and

$$
x_{00} y_{00}+y_{00} x_{00} \in A_{00} \text {. }
$$

Next we set $x=x_{11}, y=y_{00}, z=e$ in (9), (10) and (11) to obtain

$$
\begin{aligned}
(\alpha-1)\left[2 x_{11} y_{00}-e\left(x_{11} y_{00}\right)\right] & =-(2 \alpha+1)\left[\left(x_{11} y_{00}\right) e-e\left(y_{00} x_{11}\right)\right], \\
(\alpha-1)\left[\left(y_{00} x_{11}\right) e-2\left(y_{00} x_{11}\right)\right] & =(\alpha+2)\left[\left(x_{11} y_{00}\right) e-e\left(y_{00} x_{11}\right)\right], \\
(2 \alpha+1)\left[\left(y_{00} x_{11}\right) e-2 y_{00} x_{11}\right] & =-(\alpha+2)\left[2 x_{11} y_{00}-e\left(x_{11} y_{00}\right)\right],
\end{aligned}
$$

respectively. Interchanging $x$ and $y$ in (9) and making the same substitution we find

$$
\begin{aligned}
& (\alpha-1)\left[x_{11} y_{00}-y_{00} x_{11}-e\left(x_{11} y_{00}\right)\right] \\
& =-(2 \alpha+1)\left[\left(y_{00} x_{11}\right) e-y_{00} x_{11}+x_{11} y_{00}-e\left(x_{11} y_{00}\right)\right] .
\end{aligned}
$$

Multiplying (15) on the left by $e$ we have

$$
(\alpha-1) e\left(y_{00} x_{11}\right)=(2 \alpha+1)\left[e\left(y_{00} x_{11}\right) e-e\left(y_{00} x_{11}\right)\right] .
$$

Multiplying this relation on the right by $e$ we see that

$$
e\left(y_{00} x_{11}\right) e=0 \text {. }
$$

Then multiplying (13) on both sides by $e$ yields $e\left(x_{11} y_{00}\right) e=0$. Using the above substitution in (6) we find 


$$
\left(x_{11} y_{00}\right) e+\left(y_{00} x_{11}\right) e+2\left(x_{11} y_{00}\right)=e\left(y_{00} x_{11}\right)+e\left(x_{11} y_{00}\right)+2 y_{00} x_{11}
$$

and multiplication on the right by $e$ reduces this relation to $3\left(x_{11} y_{00}\right) e=\left(y_{00} x_{11}\right) e$, while multiplication on the left yields $e\left(x_{11} y_{00}\right)=3 e\left(y_{00} x_{11}\right)$. If we multiply (15) on the right by $e$ we obtain $3 \alpha\left(x_{11} y_{00}\right) e=(\alpha-1)\left(y_{00} x_{11}\right) e$ so that $\alpha\left(y_{00} x_{11}\right) e$ $=(\alpha-1)\left(y_{00} x_{11}\right) e$. Hence $\left(y_{00} x_{11}\right) e=0$. Multiplying (12) on the left by $e$ yields $(\alpha-1) e\left(x_{11} y_{00}\right)=(2 \alpha+1) e\left(y_{00} x_{11}\right)=0$ so that $e\left(y_{00} x_{11}\right)=0$. But then $\alpha e\left(y_{00} x_{11}\right)$ $=3 \alpha e\left(x_{11} y_{00}\right)=0=\alpha e\left(x_{11} y_{00}\right)$. Thus $-e\left(x_{11} y_{00}\right)=e\left(y_{00} x_{11}\right)=0$. Then (13) implies that $y_{00} x_{11}=0$ which in turn, along with (14), implies that $x_{11} y_{00}=0$.

For the elements $x_{11}, y_{10}$ we see that $\left(x_{11}, e, y_{10}\right)=0$ and setting $x=x_{11}$, $y=y_{10}, z=e$ in (9), (10), and (11) we obtain

(17) $(\alpha-1)\left[x_{11} y_{10}-e\left(x_{11} y_{10}\right)\right]=-(2 \alpha+1)\left[\left(x_{11} y_{10}\right) e+y_{10} x_{11}-e\left(y_{10} x_{11}\right)\right]$,

(18) $(\alpha-1)\left[\left(y_{10} x_{11}\right) e-2 y_{10} x_{11}\right]=(\alpha+2)\left[\left(x_{11} y_{10}\right) e+y_{10} x_{11}-e\left(y_{10} x_{11}\right)\right]$,

(19) $(2 \alpha+1)\left[\left(y_{10} x_{11}\right) e-2 y_{10} x_{11}\right]=-(\alpha+2)\left[x_{11} y_{10}-e\left(x_{11} y_{10}\right)\right]$,

respectively. Multiplying (17) on the left by $e$ we obtain $e\left(x_{11} y_{10}\right) e=0$ while multiplying (18) on the left by $e$ yields $e\left(y_{10} x_{11}\right) e=2 e\left(y_{10} x_{11}\right)$. Thus, $e\left(y_{10} x_{11}\right) e=e\left(y_{10} x_{11}\right)=0$. If we multiply (19) on the right by $e$ we find $(2 \alpha+1)\left(y_{10} x_{11}\right) e=(\alpha+2)\left(x_{11} y_{10}\right) e$, while (18) under same operation gives us $-(2 \alpha+1)\left(y_{10} x_{11}\right) e=(\alpha+2)\left(x_{11} y_{10}\right) e$. Hence, $\quad\left(x_{11} y_{10}\right) e=\left(y_{10} x_{11}\right) e=0$. The relation (18) then becomes $3 \alpha\left(y_{10} x_{11}\right)=0$. If $\alpha \neq 0$, we have $y_{10} x_{11}=0$ and (17) yields $x_{11} y_{10}=e\left(x_{11} y_{10}\right)$ or $A_{11} A_{10} \subseteq A_{10}$ and $A_{10} A_{11}=0$. If $\alpha=0$, (17) gives us $x_{11} y_{10}-y_{10} x_{11}=e\left(x_{11} y_{10}\right)$ so that $x_{11} y_{10} \in A_{10}+A_{00}, y_{10} x_{11} \in A_{00}$ and $x_{11} y_{10}-y_{10} x_{11} \in A_{10}$. In a similar fashion we find that $A_{00} A_{01} \subseteq A_{01}$ and $A_{01} A_{00}=0$ except when $\alpha=0$ and then we have $x_{00} y_{01} \in A_{01}+A_{11}, y_{01} x_{00} \in A_{11}$, $x_{00} y_{01}-y_{01} x_{00} \in A_{01}$.

Next we consider products of the form $x_{11} y_{01}, y_{01} x_{11}$. It is immediate that $\left(y_{01}, e, x_{11}\right)=0$. The substitution of $x=x_{11}, y=e, z=y_{01}$ in (9) and (10) yields

$$
\begin{aligned}
(a-1)\left[\left(x_{11} y_{01}\right) e-x_{11} y_{01}+\left(y_{01} x_{11}\right) e-y_{01} x_{11}\right] & =-(2 \alpha+1) x_{11} y_{01} \\
(\alpha-1)\left[x_{11} y_{01}-e\left(x_{11} y_{01}\right)-e\left(y_{01} x_{11}\right)\right] & =(\alpha+2) x_{11} y_{01}
\end{aligned}
$$

while setting $y=y_{01}, x=x_{11}, z=e$ in (11) gives

$$
(2 \alpha+1)\left[\left(y_{01} x_{11}\right) e-y_{01} x_{11}\right]=-(\alpha+2)\left[2 x_{11} y_{01}-e\left(x_{11} y_{01}\right)\right] .
$$

Multiplying (20) on the right by $e$ yields $\left(x_{11} y_{01}\right) e=0$, and then the same operation on (22) leads to $(\alpha+2) e\left(x_{11} y_{01}\right)=(2 \alpha+1) e\left(y_{01} x_{11}\right)$. Hence, $3 \alpha e\left(y_{01} x_{11}\right)$ $=0$. If $\alpha \neq 0$, we find $e\left(y_{01} x_{11}\right)=0=e\left(x_{11} y_{01}\right)$ and (21) implies that $x_{11} y_{01}=0$. In this case, (20) implies that $\left(y_{01} x_{11}\right) e=y_{01} x_{11}$ so that $A_{01} A_{11} \subseteq A_{01}$ and $A_{11} A_{01}=0$. If $\alpha=0$ we observe that (6) implies

$$
\left(x_{11} y_{01}+y_{01} x_{11}\right) e+x_{11} y_{01}=e\left(x_{11} y_{01}+y_{01} x_{11}\right)+y_{01} x_{11} .
$$


Multiplying this relation on the left by $e$ and simplifying we obtain $e\left(y_{01} x_{11}\right)=0$. Hence, $e\left(y_{01} x_{11}\right)=e\left(x_{11} y_{01}\right)=0$ and, as above, we have $A_{01} A_{11} \subseteq A_{01}$, $A_{11} A_{01}=0$. In a similar manner we also see that $A_{00} A_{10}=0, A_{10} A_{00} \subseteq A_{10}$.

We now consider the products $x_{10} y_{01}$ and $y_{01} x_{10}$. Clearly $\left(x_{10}, e, y_{01}\right)=\left(y_{01}, e, x_{10}\right)=0$. Setting $x=x_{10}, y=e, z=y_{01}$ in (10) and (11) yields $\left(x_{10}, y_{01}, e\right)=-\left(y_{01}, x_{10}, e\right)$ and $\left(e, x_{10}, y_{01}\right)=-\left(e, y_{01}, x_{10}\right)$. But $\left(e, x_{10}, y_{01}\right)=x_{10} y_{01}-e\left(x_{10} y_{01}\right)=e\left(y_{01} x_{10}\right)=-\left(e, y_{01}, x_{10}\right)$. Multiplying on the left by $e$, we see that $e\left(y_{01} x_{10}\right)=0$. Likewise we find that $\left(y_{01} x_{10}\right) e=0$ giving $x_{10} y_{01} \in A_{11}, y_{01} x_{10} \in A_{00}$.

Finally, we examine the terms $x_{10} y_{10}$. Setting $x=x_{10}, y=y_{10}, z=e$ in (9) we have

$$
(\alpha-1)\left[-e\left(x_{10} y_{10}\right)\right]=-(2 \alpha+1)\left[\left(x_{10} y_{10}\right) e+y_{10} x_{10}-e\left(y_{10} x_{10}\right)\right] .
$$

We multiply (23) on the left by $e$, obtaining $(\alpha-1) e\left(x_{10} y_{10}\right)=(2 \alpha+1) e\left(x_{10} y_{10}\right) e$. Then, multiplying on the right by $e$, we have $(\alpha+2) e\left(x_{10} y_{10}\right) e=0$. Thus, $e\left(x_{10} y_{10}\right) e=e\left(x_{10} y_{10}\right)=0$. Interchanging $x_{10}$ and $y_{10}$ we obtain $e\left(y_{10} x_{10}\right)=0$. Then (23) reduces to $\left(x_{10} y_{10}\right) e+y_{10} x_{10}=0$ and multiplication on the right by $e$ leads to $\left(x_{10} y_{10}\right) e=-\left(y_{10} x_{10}\right) e$. Hence, (23) becomes $\left(y_{10} x_{10}\right) e-y_{10} x_{10}=0$, giving $y_{10} x_{10}=-x_{10} y_{10} \in A_{01}$ and $x_{10}^{2}=0$. The corresponding relations hold for $x_{01}, y_{01}, x_{01}^{2}$.

Since the multiplicative relations given in Theorem 3 are the same as those satisfied in an alternative ring, it is natural to ask how close the present class of rings is to being alternative. One definition of the alternative property is that $(\sigma(x), \sigma(y), \sigma(z))=\varepsilon(\sigma)(x, y, z)$ for all $x, y, z$ in the ring and for every permutation $\sigma$, where $\varepsilon(\sigma)=1$ or -1 as $\sigma$ is even or odd. We shall now test this relation for each different way of choosing $x, y$, and $z$ belonging to $A_{11}, A_{10}, A_{01}$, and $A_{00}$ under the restrictions that $\alpha \neq 1,-\frac{1}{2},-2$ and that $A_{i i} A_{i j} \subset A_{i j}$ and $A_{i j} A_{i i}=0$ if $\alpha=0$.

Consider first the elements $x_{i i}, y_{i j}$, and $z_{j i}$ (we shall assume $i=0,1$ and $j=1-i$ for the remainder of this section). Then the relations in Theorem 3 immediately give $\left(x_{i i}, z_{j i}, y_{i j}\right)=\left(y_{i j}, x_{i i}, z_{j i}\right)=\left(z_{j i}, y_{i j}, x_{i i}\right)=0$. Substituting $x=x_{i i}, y=y_{i j}, z=z_{j i}$ in (11) yields $(2 \alpha+1)\left(y_{i j}, z_{j i}, x_{i i}\right)=-(\alpha+2)\left(z_{j i}, x_{i i}, y_{i j}\right)$. But the left side of this equation is in $A_{i i}$ while the right side is in $A_{j j}$, implying that both sides are zero. Since five of the quantities $\left(\sigma\left(x_{i i}\right), \sigma\left(y_{i j}\right), \sigma\left(z_{j i}\right)\right)$ are zero, (6) implies that the sixth one is also.

Next we see from Theorem 3 that $\left(x_{i i}, y_{i j}, z_{i i}\right)=\left(z_{i i}, y_{i j}, x_{i i}\right)=0$. If $A_{i i}$ is a subring (which can only fail when $\alpha=-1$ ), we also have $\left(y_{i j}, x_{i i}, z_{i i}\right)=\left(y_{i j}, z_{i i}, x_{i i}\right)=0$. Setting $x=y_{i j}, y=x_{i i}, z=z_{i i}$ in (11) gives $(2 \alpha+1)\left(x_{i i}, z_{i i}, y_{i j}\right)=0$, and switching $x_{i i}$ and $z_{i i}$ in this equation leads to $\left(z_{i i}, x_{i i}, y_{i j}\right)=0$. Thus $\left(\sigma\left(x_{i i}\right)\right.$, $\left.\sigma\left(y_{i j}\right), \sigma\left(z_{i i}\right)\right)=0$ for all $\sigma$ whenever $A_{i i}$ is a subring. By symmetry, we also have $\left(\sigma\left(x_{i i}\right), \sigma\left(y_{j i}\right), \sigma\left(z_{i i}\right)\right)=0$ for all $\sigma$ under the same condition.

It is again clear from Theorem 3 that $\left(x_{i i}, z_{j j}, y_{i j}\right)=\left(z_{j j}, x_{i i}, y_{i j}\right)$ 
$=\left(z_{j j}, y_{i j}, x_{i j}\right)=\left(y_{i j}, z_{j j}, x_{i i}\right)=\left(y_{i j}, x_{i i}, z_{j j}\right)=0$. The remaining permutation then follows from (6), and we have $\left(\sigma\left(x_{i i}\right), \sigma\left(y_{i j}\right), \sigma\left(z_{j j}\right)\right)=0$ for all $\sigma$. As for products of the terms $x_{i i}, y_{i i}, z_{j j}$, it is easily seen that $\left(\sigma\left(x_{i i}\right), \sigma\left(y_{i i}\right), \sigma\left(z_{j j}\right)\right)=0$ for all $\sigma$ whenever $A_{i i}$ is a subring.

There remain the cases where the associator is not necessarily zero. First we compute $\left(y_{i j}, z_{i j}, x_{i i}\right)=\left(y_{i j} z_{i j}\right) x_{i i}=-\left(z_{i j} y_{i j}\right) x_{i i}=-\left(z_{i j}, y_{i j}, x_{i i}\right)$ and $\left(x_{i i}, y_{i j}, z_{i j}\right)=\left(x_{i i} y_{i j}\right) z_{\imath j}=-z_{i j}\left(x_{i i} y_{i j}\right)=\left(z_{i j}, x_{i i}, y_{i j}\right)$. Interchanging $y_{i j}$ and $z_{i j}$ in the last equation gives $\left(x_{i i}, z_{i j}, y_{i j}\right)=\left(y_{i j}, x_{i i}, z_{i j}\right)$, and combining these with (6) gives $\left(x_{i i}, z_{i j}, y_{i j}\right)=-\left(z_{i j}, x_{i i}, y_{i j}\right)$. The substitution $x=x_{i i}, y=y_{i j}, z=z_{i j}$ in (11) now yields $(2 \alpha+1)\left[\left(y_{i j}, x_{i i}, z_{i j}\right)+\left(y_{i j}, z_{i j}, x_{i i}\right)\right]=0$. Putting together all these relations, we have $\left(\sigma\left(x_{i i}\right), \sigma\left(y_{i j}\right), \sigma\left(z_{i j}\right)\right)=\varepsilon(\sigma)\left(x_{i i}, y_{i j}, z_{i j}\right.$, for all $\sigma$. The equation $\left(\sigma\left(x_{i i}\right), \sigma\left(y_{j i}\right), \sigma\left(z_{j i}\right)\right)=\varepsilon(\sigma)\left(x_{i i}, y_{j i}, z_{j i}\right)$ for all $\sigma$ follows by symmetry.

Let us look next at associators involving the elements $x_{i j}, y_{i j}, z_{j i}$. Then $\left(x_{i j}, y_{i j}, z_{j i}\right)=\left(x_{i j} y_{i j}\right) z_{j i}=-\left(y_{i j} x_{i j}\right) z_{j i}=-\left(y_{i j}, x_{i j}, z_{j i}\right)=-z_{j i}\left(x_{i j} y_{i j}\right)$ $=\left(z_{j i}, x_{i j}, y_{i j}\right)=z_{j i}\left(y_{i j} x_{i j}\right)=-\left(z_{j i}, y_{i j}, x_{i j}\right)$. Using these relations, (6) reduces to $\left(x_{i j}, z_{j i}, y_{i j}\right)=-\left(y_{i j}, z_{j i}, x_{i j}\right)$. The substitution $x=x_{i j}, y=y_{i j}, z=z_{j i}$ in (10) now yields $(\alpha-1)\left[\left(y_{i j}, x_{i j}, z_{j i}\right)+\left(y_{i j}, z_{j i}, x_{i j}\right)\right]=0$, implying that

$$
\left(\sigma\left(x_{i j}\right), \sigma\left(y_{i j}\right), \sigma\left(z_{j i}\right)\right)=\varepsilon(\sigma)\left(x_{i j}, y_{i j}, z_{j i}\right)
$$

for all $\sigma$.

Finally we consider the elements $x_{i j}, y_{i j}, z_{i j}$. Setting $x=x_{i j}, y=y_{i j}, z=z_{i j}$ in (10) we find

$$
(\alpha-1)\left[\left(y_{i j}, x_{i j}, z_{i j}\right)+\left(y_{i j}, z_{i j}, x_{i j}\right)\right]=(\alpha+2)\left[\left(x_{i j}, y_{i j}, z_{i j}\right)+\left(z_{i j}, y_{i j}, x_{i j}\right)\right] .
$$

Then equating the components in $A_{j j}$ we have $(\alpha-1)\left[\left(y_{i j} x_{i j}\right) z_{i j}+\left(y_{i j} z_{i j}\right) x_{i j}\right]$ $=(\alpha+2)\left[\left(x_{i j} y_{i j}\right) z_{i j}+\left(z_{i j} y_{i j}\right) x_{i j}\right]$. Since $y_{i j} x_{i j}=-x_{i j} y_{i j}$ and $y_{i j} z_{i j}=-z_{i j} y_{i j}$, this equation reduces to $(2 \alpha+1)\left[\left(x_{i j} y_{i j}\right) z_{i j}+\left(z_{i j} y_{i j}\right) x_{i j}\right]=0$. But these relations are enough to imply that $\left[\sigma\left(x_{i j}\right) \sigma\left(y_{i j}\right)\right] \sigma\left(z_{i j}\right)=\varepsilon(\sigma)\left(x_{i j} y_{i j}\right) z_{i j}$ for all $\sigma$. Since, by symmetry, the same equation must hold for triples of elements from $A_{i j}$ associated the other way, we have $\left(\sigma\left(x_{i j}\right), \sigma\left(y_{i j}\right), \sigma\left(z_{i j}\right)\right)=\varepsilon(\sigma)\left(x_{i j}, y_{i j}, z_{i j}\right)$ for all $\sigma$.

Having considered all other combinations, the question of whether $A$ is alternative hinges on what happens to associators involving three elements from $A_{i i}$. We have proved all but the case $\alpha=0$ of

THEOREM 4. Let $A$ be a ring satisfying $(x, x, x)=0$ and (3) for $\alpha \neq 1$, $-\frac{1}{2},-2$, and let $A_{11}$ and $A_{00}$ be the additive subgroups corresponding to an idempotent $e$ of $A$. If $\alpha=0$ we suppose further that $A$ has no ideals $G$ such that $G^{2}=0$. Then $A$ is alternative if and only if $A_{11}$ and $A_{00}$ are alternative subrings.

To complete the proof of this theorem, we must show that $A$ satisfies $A_{i i} A_{i j} \subset A_{i j}$ and $A_{i j} A_{i i}=0$ whenever $\alpha=0$ and $A$ has no ideals $G$ satisfying $G^{2}=0$. Since $\left(x_{i i} y_{i j}-y_{i j} x_{i i}\right) \in A_{i j}$, it is sufficient to show that $A_{i j} A_{i i}=0$ under these hypotheses. Setting $G_{i}=A_{j i} A_{j j}$ and noting that $G_{i} \subset A_{i i}$, we give the proof in three lemmas. 
Lemma $1 . \quad G_{i}$ is an ideal of $A_{i i}$. Furthermore, the elements of $G_{i}$ commute with the elements of $A_{i i}$.

We begin by making the substitution $x=x_{j i}, y=z_{i i}, z=y_{j j}$ in (10) to get $-\left[\left(z_{i i}, x_{j i}, y_{j j}\right)+\left(z_{i i}, y_{j j}, x_{j i}\right)\right]=2\left[\left(x_{j i}, z_{i i}, y_{j j}\right)+\left(y_{j j}, z_{i i}, x_{j i}\right)\right]$. But since $\left(y_{j j}, z_{i i}, x_{j i}\right)=0$ and $\left(z_{i i}, x_{j i}, y_{j j}\right)=-z_{i i}\left(x_{j i} y_{j j}\right)=-z_{i i}\left(y_{j j} x_{j i}\right)=\left(z_{i i}, y_{j j}, x_{j i}\right)$, this equation reduces to $-\left(z_{i i}, x_{j i}, y_{j j}\right)=\left(x_{j i}, z_{\imath i}, y_{j j}\right)$, or

$$
z_{i i}\left(x_{j i} y_{j j}\right)=\left(x_{j i} z_{i i}\right) y_{j j}
$$

Thus, $A_{i i} G_{i} \subset G_{i}$, and $G_{i}$ is a left ideal of $A_{i i}$. On the other hand, substituting $x=y \quad y_{j i},=x_{j j}, z=z_{i i}$ in (11) yields

$$
\left[\left(x_{j i}, y_{j j}, z_{i i}\right)+\left(x_{j i}, z_{i i}, y_{j j}\right)\right]=-2\left[\left(y_{j j}, z_{i i}, x_{j i}\right)+\left(z_{i i}, y_{j j}, x_{j i}\right)\right],
$$

which may be reduced to $\left(x_{j i}, y_{j j}, z_{i i}\right)=-\left(z_{i i}, x_{j i}, y_{j j}\right)$, or $\left(x_{j i} y_{j j}\right) z_{i i}=z_{i i}\left(x_{j i} y_{j j}\right)$, using the equations above. Hence the elements of $G_{i}$ commute with the elements of $A_{i i}$, and $G_{i}$ is an ideal of $A_{i i}$.

LEMMA 2. $G_{i}^{2}=A_{j i} G_{i}=0$.

We first set $x=y_{j j}, y=x_{j i}, z=z_{j i}$ in (10) to obtain $-\left[\left(x_{j i}, y_{j j}, z_{j i}\right)+\left(x_{j i}, z_{j i}, y_{j j}\right)\right]=2\left[\left(y_{j j}, x_{j i}, z_{j i}\right)+\left(z_{j i}, x_{j i}, y_{j j}\right)\right]$. Equating the $A_{j i}$ components of this equation gives $x_{j i}\left(z_{j i} y_{j j}\right)=-2 z_{j i}\left(x_{j i} y_{j j}\right)$. But switching $x_{j i}$ and $z_{j i}$ in the last equation gives $z_{j i}\left(x_{j i} y_{j j}\right)=-2 x_{j i}\left(z_{j i} y_{j j}\right)$, and subtracting twice this from the previous equation gives $3 x_{j i}\left(z_{j i} y_{j j}\right)=0$, or $x_{j i}\left(z_{j i} y_{j j}\right)=0$. This implies that $A_{j i} G_{i}=0$. To show that $G_{i}^{2}=0$, it is sufficient to prove that $\left(z_{j i} w_{j j}\right)\left(x_{j i} y_{j j}\right)=0$. But setting $z_{i i}=z_{j i} w_{j j}$ in (23) gives $\left(z_{j i} w_{j j}\right)\left(x_{j i} y_{j j}\right)$ $=\left(x_{j i}\left(z_{j i} w_{j j}\right)\right) y_{j j}=0$, since $x_{j i}\left(z_{j i} w_{j j}\right)=0$ from the last paragraph.

The proof of Theorem 4 will be complete when we have established

LemMA 3. Let $G=G_{1}+G_{0}$. Then $G$ is an ideal of $A$ and $G^{2}=0$.

To prove that $G$ is an ideal of $A$, it is sufficient to show that $A G_{i}+G_{i} A \subset G$. But $A_{j j} G_{i}=G_{i} A_{j j}=G_{i} A_{j i}=0$ by Theorem $3, A_{j i} G_{i}=0$ and $A_{i i} G_{i}=G_{i} A_{i i} \subset G_{i}$ by the two previous lemmas, and $A_{i j} G \subset A_{i j} A_{i i}=G_{j}$. Thus we need only prove that $G_{i} A_{i j} \subset G$. For this we add together (10) and (6) and set $x=x_{j i}, y=y_{j j}$, $z=z_{i j}$ to obtain

$$
0=\left(x_{j i}, y_{j j}, z_{i j}\right)+\left(z_{i j}, y_{j j}, x_{j i}\right)-\left(x_{j i}, z_{i j}, y_{j j}\right)-\left(z_{i j}, x_{j i}, y_{j j}\right) .
$$

Now $\left(x_{j i}, y_{j j}, z_{i j}\right)=\left(x_{j i} y_{j j}\right) z_{i j} \in G_{i} A_{i j} \subset A_{i j}+G_{j}$, while none of the other associators in this equation has a nonzero component in $A_{i j}$, since $\left(z_{i j}, y_{j j}, x_{j i}\right)$ $=\left(z_{i j} y_{j j}\right) x_{j i}-z_{i j}\left(y_{j j} x_{j i}\right) \in A_{i j} A_{j i}+A_{i j}\left(A_{j i}+G_{i}\right) \subset A_{i i}+G_{j},\left(x_{j i}, z_{i j}, y_{j j}\right)$ $=\left(x_{j i} z_{i j}\right) y_{j j}-x_{j i}\left(z_{i j} y_{j j}\right) \in A_{j j} A_{j j}+A_{j i} A_{i j} \subset A_{j j}$, and $\left(z_{i j}, x_{j i}, y_{j j}\right)$ $=-z_{i j}\left(x_{j i} y_{j j}\right) \in A_{i j} G_{i} \subset G_{j}$. Thus $\left(x_{j i} y_{j j}\right) z_{i j} \in G_{j}$, which implies that 
$G_{i} A_{i j} \subset G_{j} \subset G$. And finally, $G^{2}=0$ follows immediately from $G_{i}^{2}=0$ and $G_{i} G_{j} \subset A_{i i} A_{j j}=0$ to complete the proof.

We shall show next that under a suitable additional hypothesis the $A_{i i}$ are alternative subrings of $A$. We first prove

Lemмa 4. Under the hypotheses of Theorem 4,

$$
L=A_{10} A_{01}+A_{10}+A_{01}+A_{01} A_{10}
$$

is an ideal of $A$.

We see from the multiplicative relations for the $A_{k l}$ 's that it will suffice to show that $A_{i i}\left(A_{i j} A_{j i}\right)+\left(A_{i j} A_{j i}\right) A_{i i} \subset A_{i j} A_{j i}$. But this follows immediately from the relations $\left(x_{i i}, y_{i j}, z_{j i}\right)=\left(y_{i j}, z_{j i}, x_{i i}\right)=0$, which were derived in the proof of the last theorem. This lemma has the

COROLlaRY. If $A$ is a simple ring satisfying the hypotheses of Theorem 4, then either $A_{10}+A_{01}=0$, or $A_{11}=A_{10} A_{01}$ and $A_{00}=A_{01} A_{10}$.

Lemma 5. If $A_{i i}=A_{i j} A_{j i}$, then $A_{i i}$ is an associative subring.

To show that $A_{i i}$ is a subring under this hypothesis, it is sufficient to remark that the product $x_{i i}\left(y_{i j} y_{j i}\right)=\left(x_{i i} y_{i j}\right) y_{j i}$ is in $A_{i i}$. The associativity of $A_{i i}$ follows from the calculation $\left(x_{i i}\left(y_{i j} y_{j i}\right)\right) z_{i i}=\left(\left(x_{i i} y_{i j}\right) y_{j i}\right) z_{i i}=\left(x_{i i} y_{i j}\right)\left(y_{j i} z_{i i}\right)=x_{i i}\left(y_{i j}\left(y_{j i} z_{i i}\right)\right)$ $=x_{i i}\left(\left(y_{i j} y_{j i}\right) z_{i i}\right)$. These lemmas prove

THeORem 5. Let $A$ be a simple ring satisfying $(x, x, x)=0$ and (3) for $\alpha \neq 1,-\frac{1}{2},-2$. Suppose further that $A$ has an idempotent $e$ such that $A_{10}+A_{01} \neq 0$. Then $A$ is an alternative ring.

If we exclude the case $\alpha=-1$, we may restate this theorem in a little stronger form. For then $A_{10}+A_{01}=0$ implies $A=A_{11} \oplus A_{00}$, in which case either $A_{00}$ is a nontrivial ideal or $e$ is the unity element of $A$.

THEOREM 6. Let $A$ be a simple ring with idempotent $e$ satisfying $(x, x, x)=0$ and (3) for $\alpha \neq 1,-\frac{1}{2},-1,-2$. Then either $A$ is alternative or $e$ is the unity element.

An example to show that this theorem is not true for $\alpha=-1$ may be found in [9].

It might be well to point out at this juncture some of the known results for the special cases $\alpha=1,-\frac{1}{2},-2$. The cases $\alpha=-\frac{1}{2}$ and $\alpha=-2$ correspond to the left alternative and right alternative rings respectively which are anti-isomorphic while $\alpha=1$ corresponds to the flexible rings. Albert [3] has shown that any semisimple finite dimensional right al ternative algebra of characteristic not 2 is alternative. In [10], Maneri has proved that any simple right alternative ring of characteristic not 2 or 3 that satisfies (5) and has an idempotent $e \neq 1$ is alternative. Oehmke $[10]$ has shown that any simple power-associative flexible ring (characteristic not 2 
or 3) with two orthogonal idempotents whose sum is not 1 is a noncommutative Jordan algebra [13]. He also proved under the additional hypothesis that $A$ is a finite dimensional algebra (characteristic not 2,3 , or 5 ) that $A$ is one of the following:

(i) a commutative Jordan algebra,

(ii) a quasi-associative algebra,

(iii) an algebra of degree 1 or 2 .

3. Semi-simple algebras. Throughout this section $A$ will be a finite-dimensional power-associative algebra over a field $F$ of characteristic not 2 or 3 which satisfies (3) with $\alpha \neq-1$. Since $A$ is power-associative, it must necessarily satisfy $(x, x, x)=0$, so that the results of the preceding section may be applied.

THEOREM 7. Let $A$ be a simple power-associative algebra satisfying (3) with $\alpha \neq-1$. Then $A$ has a unity element.

If $\alpha=1$, then (6) and (3) imply the flexible law, and this result follows from [11]. If $\alpha=-\frac{1}{2}$ or -2 , then (6) and (3) imply the left or right alternative law, respectively, and it is again known that $A$ has a unity element [3]. For the remaining values of $\alpha$, this theorem follows from Theorem 6 with the remark that a simple alternative algebra has a unity element.

Next, we define the radical $N$ of $A$ to be the maximal nil ideal of $A$, and we call $A$ semi-simple whenever $N=0$. We are now ready for the two main theorems of this section.

THEOREM 8. Let $A$ be a power-associative algebra satisfying (3) with $\alpha \neq 1,-\frac{1}{2},-1,-2$. Then for any principal idempotent $e$ of $A$, $A_{10}+A_{01}+A_{00} \subset N$.

THEOREM 9. Let A be a semi-simple power-associative algebra satisfying (3) with $\alpha \neq-1$. Then $A$ has a unity element and is the direct sum of simple algebras.

Theorem 9 is already known for $\alpha=1$ [11] and $\alpha=-\frac{1}{2},-2$ [3]. For the remaining values of $\alpha$ we shall prove both theorems simultaneously with an induction on the dimension $n$ of $A$. The desired results are immediate when $n=1$. If $A$ is not semi-simple and if $e$ is a principal idempotent of $A$, then $e+N$ is a principal idempotent of $A / N=B$ and $B_{10}+B_{01}+B_{00}$ (with respect to $e+N)$ is the image of $A_{10}+A_{01}+A_{00}$. But since $B$ is semi-simple of smaller dimension, $B_{10}+B_{01}+B_{00}=0$ and $A_{10}+A_{01}+A_{00} \subset N$.

There remains the case when $A$ is semi-simple. If $A$ is simple, both theorems follow from Theorem 7. Otherwise, $A$ contains a proper non-nil ideal $D$, and $D$ contains a principal idempotent $e$. Decomposing $D$ with respect to $e$ we have $D=D_{11}+D_{10}+D_{01}+D_{00}$. But since $e \in D$, we then see that the decomposition of $A$ is $A=D_{11}+D_{10}+D_{01}+A_{00}$ where $D_{00} \subset A_{00}$. Letting $M$ be the radical 
of $D$, we have $D_{10}+D_{01}+D_{00} \subset M$ by the inductive hypothesis. We claim that $M$ is also a nil ideal of $A$. For, since $D_{00} A_{00}+A_{00} D_{00} \subset D \cap A_{00}=D_{00} \subset M$, we may calculate

$M A+A M \subset M+M A_{00}+A_{00} M \subset M+D_{10}+D_{01}+D_{00} A_{00}+A_{00} D_{00}=M$.

Thus $M=0, D_{10}+D_{01}+D_{00}=0$, and $A=D \oplus A_{00}$. Both theorems now follow by applying the inductive hypotheses to $D$ and $A_{00}$.

\section{BIBLIOGRAPHY}

1. A. A. Albert, Power-associative rings, Trans. Amer. Math. Soc. 64 (1948), 552-597.

2. - Almost alternative algebras, Portugaliae Math. 8 (1949), 23-36.

3. - - Structure of right alternative algebras, Ann. of Math. (2) 59 (1954), 407-417.

4. Erwin Kleinfeld, Simple alternative rings, Ann. of Math. (2) 58 (1953), 544-547.

5. - Simple algebras of type $(1,1)$ are associative, Canad. J. Math. 13 (1961), 129-148.

6. - Associator dependent rings, Arch. Math. 13 (1962), 203-212.

7. L. A. Kokoris, A class of almost alternative algebras, Canad. J. Math. 8 (1956), 250-255.

8. - On rings of $(\gamma, \delta)$-type, Proc. Amer. Math. Soc. 9 (1958), 897-904.

9. Frank Kosier, On a class of nonflexible algebras, Trans. Amer. Math. Soc. 102 (1962), 299-318.

10. Carl Maneri, Simple $(-1,1)$ rings with an idempotent, Proc. Amer. Math. Soc. 14 (1963), $110-117$.

11. Robert Oehmke, On flexible algebras, Ann. of Math. (2) 68 (1958), 221-250.

12. D. Rodabaugh, A generalization of the flexible law, Ph. D. thesis, Illinois Institute of Technology, 1963.

13. R. D. Schafer, Alternative algebras over an arbitrary field, Bull. Amer. Math. Soc. 49 (1943), 549-555.

14. - Noncommutative Jordan algebras of characteristic 0, Proc. Amer. Math. Soc. 6 (1955), 472-475.

15. - On a class of quadratic algebras, Proc. Amer. Math. Soc. 13 (1962), 187-191.

SYRACUSE UNIVERSITY,

SYRACUSE, New York

UNIVERSITY OF WISCONSIN,

MADISON, Wisconsin

ILLINOIS INSITITUTE OF TECHNOLOGY,

ChicAGo, IllinoIS 